\title{
Molecular Dynamics Study of Grain Boundary Diffusion of Hydrogen in Tungsten
}

\author{
U. von Toussaint ${ }^{1}$, S. Gori ${ }^{1}$, A. Manhard ${ }^{1}$, T. Höschen ${ }^{1}$, \\ C. Höschen ${ }^{2}$ \\ ${ }^{1}$ Max-Planck-Institut für Plasmaphysik, EURATOM Association, Boltzmannstr. 2, 85748 \\ Garching, Germany \\ ${ }^{2}$ Chair of Soil Science, Department of Ecology and Ecosystem Management, Technical \\ University of Munich, Emil-Ramann-Str. 2, 85354 Freising, Germany \\ E-mail: udo.v.toussaint@ipp.mpg.de
}

\begin{abstract}
Understanding the influence of the microstructure of tungsten on hydrogen transport is crucial for the use of tungsten as first-wall material in fusion reactors. Here we report results from molecular dynamics and transition state studies on the influence of grain boundaries in tungsten on the transport of hydrogen. An exhaustive mapping of possible minimum activation energy migration trajectories for hydrogen as trace impurity reveals a strongly modified activation energy distribution in the neighborhood of grain boundaries together with an altered connectivity matrix. The results indicate that grain boundaries in polycrystalline tungsten may provide an important transport channel, especially for neutron damaged tungsten.
\end{abstract}

PACS numbers: $52.40 . \mathrm{Hf}, 61.72 . \mathrm{Mm}, 66.30 . \mathrm{J}-$, 89.30.Jj

Submitted to: Phys. Scr. / PFMC-13

\section{Introduction}

Tungsten is presently considered as one of the most favorable materials for plasma-facing components in future fusion reactors $[1,2]$. However, as plasma facing material tungsten will be exposed to high fluxes of tritium, alpha-particles and neutrons which may change the material behavior. From a safety point of view tritium retention and tritium transport are the most crucial aspects. Due to the low solubility of hydrogen in tungsten [3] the retention of tritium in undamaged tungsten is considered as uncritical. The situation may be different if high-energy neutrons create additional trap sites in the material. However, assuming a bulk trap-diffusion model for hydrogen propagation it was estimated that the tritium diffusion rate is low and the subsequent (slow) saturation of tungsten within tolerable limits [4]. This estimate, however, does not take into account the structural anisotropy (i.e. grain boundaries) of tungsten used as plasma facing material which may alter the permeation properties. A detailed understanding and correct model building of the transport properties of hydrogen in tungsten is therefore mandatory for reliable extrapolations towards a fusion reactor. 


\section{Motivation}

Essentially there are four distinct mechanisms which may contribute to the effective diffusion (permeation) rate of hydrogen in tungsten:

- bulk diffusion

- trapping

- grain boundary diffusion

- surface recombination

Under fusion relevant flux conditions the influence of the last item, surface recombination, is considered to be negligible [5]. The majority of the theoretical work on hydrogen transport in tungsten is addressing the first and second item based on zero or one-dimensional diffusion models (see e.g. [6] and references therein) of different complexity. The typical model uses trap energy distributions - commonly derived from TDS measurements - and applies a depth-dependent trap-diffusion model to capture various effects (e.g. altered trap energy distributions due to ion bombardment) to derive the effective diffusion rates. Although this is a very sensible approach to describe the transport in homogeneous samples it is by no means certain that those phenomenological models are adequate to describe the transport in locally inhomogeneous environments like polycrystalline metals. The influence of grain boundaries on the diffusion of hydrogen has been experimentally studied in several technologically important materials such as, e.g., Nickel $([7,8])$, stainless steel [9] or palladium [10], often driven by the observation of rapid hydrogen embrittlement [11]. Similar experiments on tungsten have apparently only very rarely been conducted. This is probably related to the much lower equilibrium hydrogen concentrations in tungsten which complicates the use of diagnostic techniques (e.g. silver decoration [12]). However, recent measurements on polycrystalline tungsten using a secondary ion mass spectroscopy device (Cameca NanoSIMS 50L) with a lateral resolution of up to $50 \mathrm{~nm}$ at the Technical University of Munich indicate the possible enrichment of hydrogen along grain boundaries (see Figure 1). Similar enrichment of hydrogen along grain boundaries in tungsten has been suggested as a mechanism for grain ejection under hydrogen bombardment [13].

\section{Modeling Details}

The interaction of hydrogen with tungsten has been calculated based on a classical many-body bond-order potential [14] $V=V\left(\mathbf{r}_{1}, \ldots, \mathbf{r}_{N}\right)$ with analytical first derivatives. Since at elevated temperatures above room temperature the migration of hydrogen in bcc metals is presumably well described by classical rate theory [15] the jump rate $\Gamma$ can be expressed by

$$
\Gamma=\frac{k_{b} T}{h} \frac{Z_{S}^{*}}{Z_{\text {Min }}}
$$

where $Z_{\text {Min }}$ is the partition function at the minimum, and $Z_{S}^{*}$ is the partition function at the saddle point for all normal coordinates except in the direction of the reaction coordinate [16]. Under the assumption that the lattice vibrations of the heavy tungsten 
atoms are sufficiently decoupled from the lighter hydrogen isotopes during a transition of the hydrogen atom [17] the contributions of the metal atoms to the respective partition functions cancel each other. The evaluation of the partition functions in harmonic approximation (assuming $\hbar \omega<<k_{b} T$ ) then results in the well known classical expression [18]

$$
\Gamma_{C}=\frac{\prod_{i=1}^{3} \nu_{i}^{M i n}}{\prod_{i=1}^{2} \nu_{i}^{S}} \exp \left(-\frac{\left(E_{S}-E_{M i n}\right)}{k_{b} T}\right),
$$

where $\nu_{i}^{M i n}$ and $\nu_{i}^{S}$ correspond to the vibration frequency of the hydrogen atom at the minimum and saddle point, respectively. The height of the activation barrier $E_{\text {act }}=\left(E_{S}-E_{\text {Min }}\right)$ between the energy at the minimum $E_{\text {Min }}$ and at the saddle point derived from experiments is in the range of $0.39-1.07 \mathrm{eV}[3,19]$ for jumps between interstitial sites.

However, this classical treatment neglects the fact that the energy levels of the localized vibration of a proton fulfill $h \nu \gg k_{b} T$ in bcc metals at room temperature. This can be taken into account (to first order) by using the quantum mechanical solution of the vibrational partition function,

$$
Z_{v i b}=\sum_{n=0}^{\infty} \exp \left(-\frac{h \nu(n+1 / 2)}{k_{b} T}\right)=\frac{\exp \left(-\frac{h \nu}{2 k_{b} T}\right)}{1-\exp \left(-\frac{h \nu}{k_{b} T}\right)}=\frac{1}{2 \sinh \left(h \nu / 2 k_{b} T\right)}
$$

The jump rate Equation 1 can then be expressed as modification of the classical jump rate $\Gamma_{C} \ddagger$

$$
\Gamma=\frac{\prod_{i=1}^{3} \frac{\sinh \left(h \nu_{i}^{M i n} / 2 k_{b} T\right)}{h \nu_{i}^{M i n} / 2 k_{b} T}}{\prod_{i=1}^{2} \frac{\sinh \left(h \nu_{i}^{S} / 2 k_{b} T\right)}{h \nu_{i}^{S} / 2 k_{b} T}} \Gamma_{C}
$$

Here the prefactor is temperature dependent, in contrast to the classical solution given in Equation 2. Based on Equation 4 the hydrogen jump rate can be derived from the energy values and the eigenvalues of the Hessian matrix (providing the vibrational frequencies $\nu_{i}$ in harmonic approximation) of the potential at the minimum and first order saddle point locations of the hydrogen atoms.

While locating (local) potential energy minima can routinely and effectively be done using, e.g., conjugate gradient methods [21] finding saddle points on potential-energy surfaces can be extremely difficult [22]. A point $\mathbf{x}^{*}$ is a first order saddle point of a function $f(\mathbf{x})$ provided $\left.\nabla f(\mathbf{x})\right|_{\mathbf{x}^{*}}=0$ and, in addition, exactly one eigenvalue of the Hessian matrix of $f$ evaluated at $\mathbf{x}^{*}$ has to be negative while all the other eigenvalues are positive.

Several algorithms have been developed, the nudged-elastic band method [23], the growing-string method [24], the partitioned rational function optimization method [25] or the dimer method [26]. However, even using the best performing dimer method [22] we could not extract the complete set of possible reaction pathways in test cases (see

$\ddagger$ Please note that in the corresponding Equations (8.41-8.42) in the well-known book 'Hydrogen in Metals I' by G. Alefeld and J. Völkl [15] a factor of two appears to be missing. Compare e.g. [20]. 
below). For that reason we implemented a different approach based on an exhaustive minimum search $H\left(\mathbf{r}_{1}, \ldots, \mathbf{r}_{N}\right)=0$ of the function

$$
H\left(\mathbf{r}_{1}, \ldots, \mathbf{r}_{N}\right)=(\nabla V)^{T}(\nabla V) \text {. }
$$

which maps the vector requirement $\nabla V=0$ into a minimization problem of a scalar function. For all identified minima $\mathbf{x}^{*}$ of $H$ the corresponding eigenvalues of the Hessian matrix of $V$ have been computed and the first order saddle points have been identified. Subsequently, starting from each of the identified saddle points the reaction (transition) paths to the corresponding minima sites are computed. In summary, the presented approach provides a list of minima locations and energies, the connectivity matrix (which of the neighboring minima can be reached within a single transition step from a given minimum) and the transition rates.

\section{Model Validation}

The effective transport velocity within a network of different propagation speeds is dominated by the fasted migration paths. Therefore, any algorithm for the computation of migration paths should be scrutinized if it really detects all relevant saddle points within a sample. One of the test cases we used for the algorithm was a perfect tungsten crystal with bcc structure - the high symmetry facilitates the detection of transition paths missed by the algorithm. Within the sample the search for minima of $H$ was started from a 3-D grid with $0.1 \AA$ spacing between neighboring grid points. The result is displayed in Figure 2 where the identified minima of the potential energy are indicated by squares, and circles represent the identified saddle points. A solid line gives the reaction pathway between to minima. The symmetry of the derived migration network indicates that no migration paths have been missed. Other algorithms (NEB, dimer method) have also been tried. However, despite the higher efficiency of these algorithms in terms of number of required potential evaluations to reach a saddle point, it turned out that it is almost impossible to compile a complete list of saddle points in irregular test structures because the algorithms repeatedly converged to the same saddle point instead of detecting neighboring ones.

In Figure 3 the tetrahedral sites of a bcc lattice are indicated by solid circles. Comparing Figure 3 and Figure 2 it is obvious that the computed migration paths correspond to transitions from tetrahedral interstitial positions to neighboring ones. Other suggested migration paths i.e. from a tetrahedral interstitial positions to an octahedral interstitial position have not been observed as first order transitions. This result is supported by several recent density functional theory studies [17, 27, 28, 29] which also conclude that the energetically preferred migration path of hydrogen in single crystalline tungsten is via tetrahedral interstitial positions - although the derived activation energies in these DFT computations differ by almost 100\%. Experimentally, ion-channeling experiments found that interstitial hydrogen in single-crystal tungsten sits at tetrahedral sites [30] - a result which is also in favor of a migration via tetrahedral interstitial positions. 


\section{Results}

Whereas the experimental and modeling results on single crystalline tungsten are in good agreement, only very little is known about the migration along grain boundaries in tungsten. Nevertheless, grain boundaries will be present in tungsten used as a plasmafacing material - either from the very beginning or due to recrystallisation of amorphous tungsten during operation of the fusion device. In Figure 4 a transmission electron micrograph of a grain boundary in recrystallized tungsten is displayed. The relative orientations of the tungsten grains on the left and right of the vertical grain boundary can be deduced from the respective pattern orientations. Please note the very narrow horizontal extension of the grain boundary. To model such a grain boundary the upper half of an MD single crystal tungsten sample was rotated around the vertical axis by 20 degrees and subsequently tempered at $1000 \mathrm{~K}$ for 1 ns to relax the artificially created interface. The resulting sample is shown in Figure 5. The upper and lower half of the sample still exhibit the underlying crystal structure (with small deviations due to thermal fluctuations) but at the interface the altered (relaxed) atom positions with respect to the ideal bcc structure can clearly be seen. For this interface the saddle points, minima and reaction paths have been calculated. Figure 6 shows a detail of the 3-D minimum activation energy migration paths of hydrogen at the grain boundary in tungsten. By comparison with Figure 2 it is evident that the locations of the minimum energy sites as well as the connectivity pattern have changed. The connectivity matrix is given in Figure 7. Please note that by definition this matrix is symmetric. The pattern reveals that the network of possible migration paths is local (because the matrix elements different from zero are all centered around the diagonal) but considerably less regular as the one for the single crystal sample. An analysis of activation energies for hydrogen migration in the grain boundary leads to the energy distribution shown in Figure 8. The distribution is asymmetric with a tail extending to high activation energies of up to $4 \mathrm{eV}$. At the same time migration paths with very low activation energies are present in the grain boundary, possibly providing fast transport channels. However, the net effect on the overall migration of hydrogen depends on the spatial pattern of these channels: fast but spatially isolated channels will hardly affect the average migration speed. A quantitative evaluation of the hydrogen migration by stochastic simulation $[31,21]$ is feasible solely based on the previously derived connectivity information and the individual transition rates given by Equation 4 . Please note that the transition rate $\Gamma_{i \rightarrow j}$ from minimum site $i$ to minimum site $j$, differs from $\Gamma_{j \rightarrow i}$ in general. The mesoscopic diffusion rates in the grain boundary derived from stochastic simulations fluctuate by two orders of magnitude around the tungsten bulk value, displaying a kind of Moiré pattern within the two-dimensional grain boundary. On average the effective diffusion rate of the analyzed grain boundary exceeds the bulk value by a factor of five, thus demonstrating that grain boundaries may act as fast transport channels. This factor may be even higher when the bulk diffusivity of tungsten is reduced, e.g. due to additional trap sites caused by neutron induced damage. However, due to the fluctuations of the results even on a nanometer scale the sample size has to be enlarged to obtain less fluctuating values. In addition it also reveals that an isolated consideration of changes of individual activation barriers may be misleading for inhomogeneous samples. 


\section{Conclusions and Outlook}

An approach - based on a coupling of molecular dynamics simulations and harmonic transition state theory - has been presented that enables the computation of local and effective diffusion rates in spatially inhomogeneous samples, i.e. for grain boundaries. It was shown that the network of migration paths and corresponding activation energies in grain boundaries may be very different from the situation in single crystal tungsten, possibly leading to enhanced diffusion rates for hydrogen (here a factor of five) in the trace impurity limit.

Although the presented approach already provides a means towards a better understanding of the influence of grain boundaries on the effective transport it appears worthwhile to study the influence of two further extensions on the transport of hydrogen:

- consideration of the influence of thermal movement of the lattice atoms on the activation energies using a series of MD snapshots as samples

- taking finite hydrogen concentration into account, e.g. by filling the deepest traps with hydrogen - this will alter the transport network.

This is work presently in progress. However, especially the second extension is computationally very expensive. 


\section{References}

[1] Bolt H, Barabash V, Federici G, Linke J, Loarte A, Roth J and Sato K 2002 J. Nucl. Mater. 307-311 43-52

[2] Kaufmann M and Neu R 2007 Fusion Eng. Des. 82521

[3] Frauenfelder R 1969 J. Vac. Sci. Technol. 6388

[4] Lipschultz B, Roth J, Davis J, Doerner R, Haasz A and Kallenbach A 2010 Plasma science and fusion center report PSFC/RR-10-4 Massachusetts Institute of Technology

[5] Causey R, Wilson K, Venhaus T and Wampler W 1999 Journal of Nuclear Materials 266-269 467-471

[6] Causey R A, Brooks J N and Federici G 2002 Fusion Eng. Des. 61-62 525-536

[7] Brass A and Chanfreau A 1996 Acta Metallurgica 44 3823-3831

[8] Doyle D, Palumbo G, Aust K, El-Sherik A and Erb U 1995 Acta Metall. Mater. 43 3027-3033

[9] Abraham P, Elleman T and Verghese K 1978 Journal of Nuclear Materials 73 77-88

[10] Mütschele T and Kirchheim R 1987 Scripta Metallurgica 21135 - 140

[11] Jones R 1990 Acta metall. mater. 38 1703-1718

[12] Yao J and Cahoon J 1991 Acta metall. mater. 39 119-126

[13] Ueda Y, Kashiwagi H, Fukumoto M, Ohtsuka Y and Yoshida N 2009 Fusion Science and Technology 56 85-90

[14] Juslin N, Erhart P, Träskelin P, Nord J, Henriksson K, Nordlund K, Salonen E and Albe K 2005 Journal of Applied Physics $\mathbf{9 8} 123520$

[15] Kehr K 1978 Theory of the diffusion of hydrogen in metals Hydrogen in Metals I ed Alefeld G and Völkl J (Berlin: Springer) pp 197-226

[16] Eyring H 1935 J. Chem. Phys. 3107

[17] Johnson D and Carter E 2010 J. Mater. Res. 25 315-327

[18] Vineyard G 1957 J. Phys. Chem. Solids 3121

[19] Zakharov A, Sharapov V and Evko E 1973 Fiz. Khim. Mekh. Mater 929

[20] Katz L, Guinan M and Borg R J 1971 Phys. Rev. B 4 330-341

[21] Press W, Teukolsky S, Vetterling W and Flannery B 2007 Numerical Recipes 3rd ed (Cambridge: Cambridge University Press)

[22] Heyden A, Bell A and Keil F 2005 J. Chem. Phys. 123224101

[23] Henkelman G and Jonsson H 2000 J. Phys. Chem. 1139978

[24] Peters B, Heyden A, Bell A and Chakraborty A 2004 J. Phys. Chem. 1207877

[25] Banerjee A, Adams N, Simons J and Shepard R 1985 J. Phys. Chem. 8952

[26] Henkelman G and Jonsson H 1999 J. Phys. Chem. 1117010

[27] Liu Y L, Zhang Y, Luo G N and Lu G 2009 Journal of Nuclear Materials 390-391 1032-1034

[28] Xu J and Zhao J 2009 Nucl. Instrum. Meth. B 267 3170-3174

[29] Heinola K and Ahlgren T 2010 Journal of Applied Physics 107113531

[30] Picraux S and Vook F 1974 Phys. Rev. Lett. 331216

[31] Gillespie D 1976 J. Comp. Physics 11 403-434

[32] Fukai Y 1993 The Metal-Hydrogen System (Berlin: Springer) page 189, Fig. 4.59 


\section{Figures}

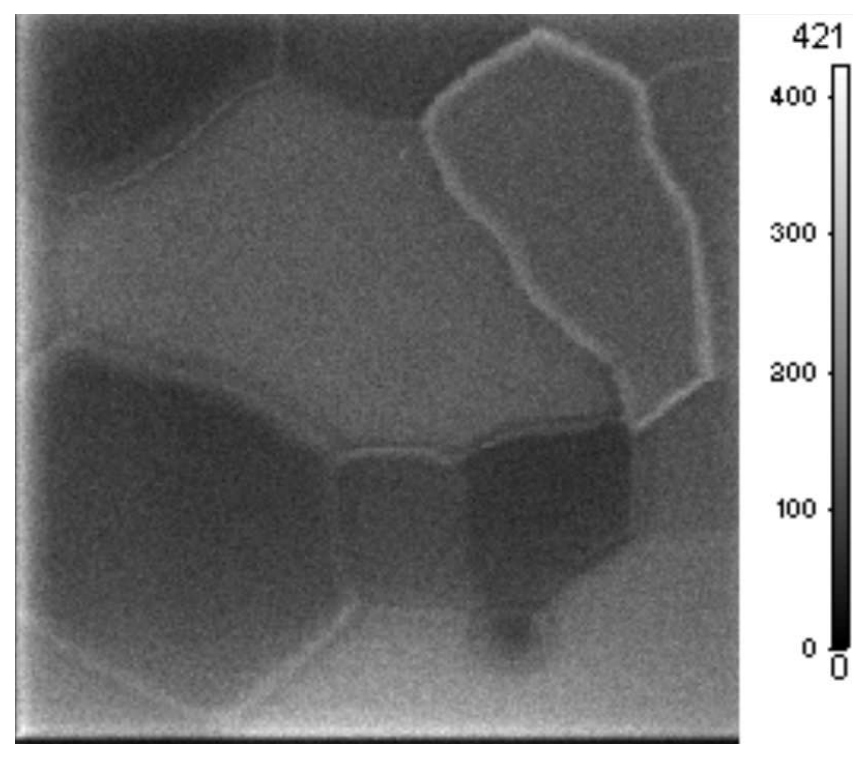

Figure 1. Hydrogen signal measured by Nano-SIMS on a recrystallized tungsten sample as grey-scale image based on the number of recorded events (darker color corresponds to less hydrogen). To improve the signal-to-noise ratio the displayed image is the superposition of 100 individual recordings with a beam current of $1.3 \mathrm{pA}$. The image size is $30 \times 30 \mu \mathrm{m}^{2}$ and its resolution is $256 \times 256$ pixel with a total measurement time of $100 \mathrm{~ms} /$ pixel. The decoration of the grain boundaries, especially in the upper right of the image is clearly visible. The data for all other recorded species (e.g. oxygen or carbon) do not exhibit this feature, thus reducing the likelihood for a geometric artifact.

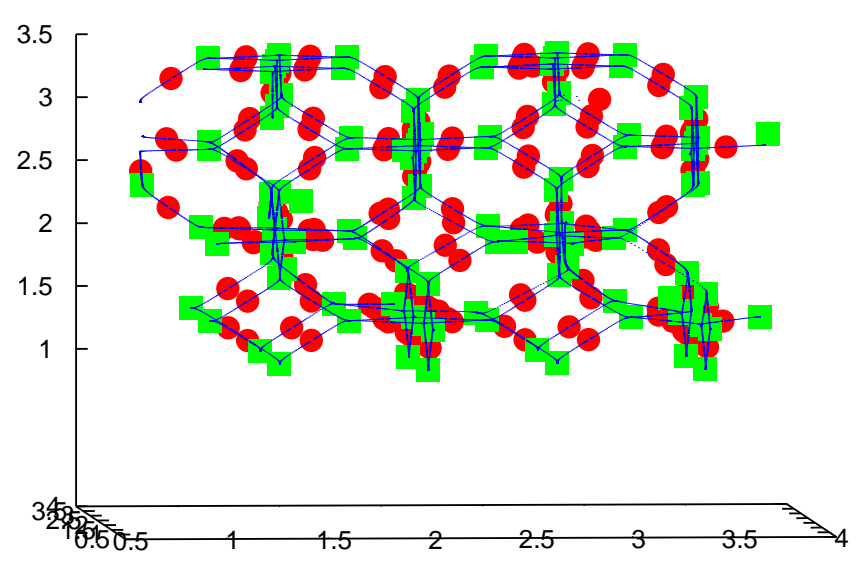

Figure 2. 3-D minimum activation energy migration paths of hydrogen in an perfect bcctungsten single crystal in perspective view (lenght units are $\AA$ ). The squares indicate the minimum energy sites of hydrogen in the lattice and the circles represent the position of the saddle points. The solid lines show the network of minimum activation energy migration paths of hydrogen atoms in the tungsten lattice. By comparison with figure 3 it is evident that the minimum energy sites coincide with the tetrahedral sites in the lattice. However, the minimum activation energy migration paths are not straight lines but exhibit some curvature. 


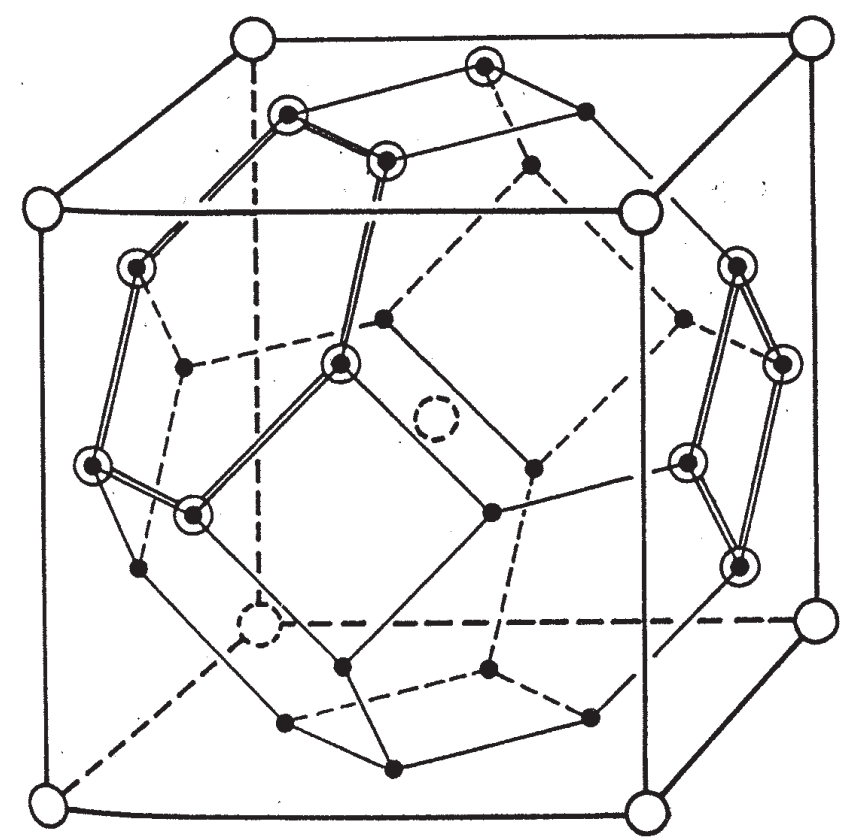

Figure 3. Schematic drawing of a bcc-crystal cell. The open circles (solid and dashed line style) represent tungsten atoms, the solid circles indicate the tetrahedral sites, the preferred sites for interstitial hydrogen in an undistorted lattice. Lines are to guide the eye (adapted from [32]).

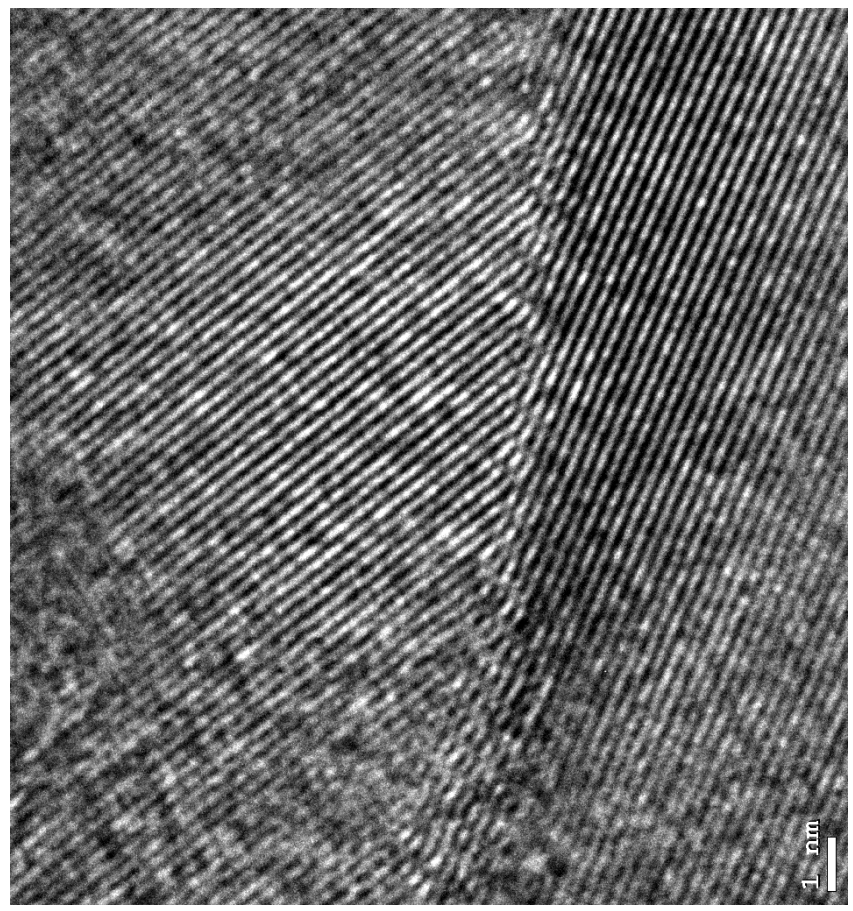

Figure 4. High resolution transmission electron micrograph of a grain boundary in recrystallized tungsten. The relative orientations of the tungsten grains on the left and on the right of the vertical grain boundary can be deduced from the different oriented patterns. 


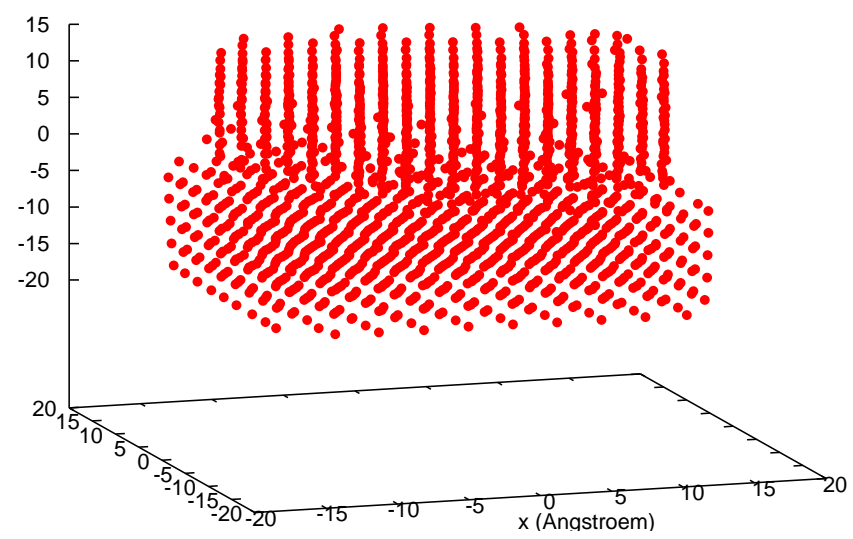

Figure 5. Positions of tungsten atoms in the molecular dynamics sample used to compute the saddle points after relaxation. The upper half of a perfect bcc tungsten crystal has been rotated by 20 degrees around the vertical axis with respect to the lower half. The resulting sample has subsequently been relaxed by tempering at $1000 \mathrm{~K}$ for $1 \mathrm{~ns}$.

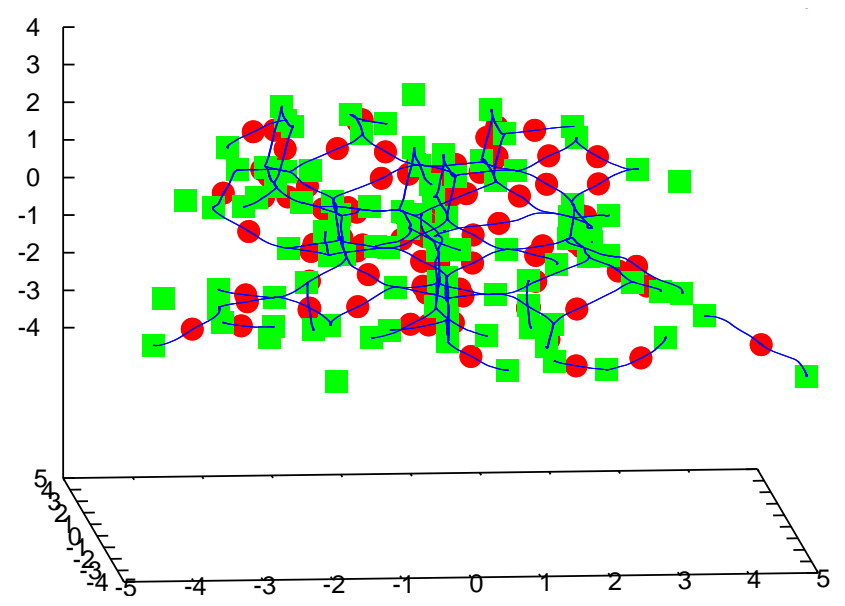

Figure 6. 3-D minimum activation energy migration paths of hydrogen at an annealed interface between two perfect bcc tungsten single crystals, rotated by 20 degrees with respect to each other in perspective view. The squares indicate the minimum energy sites of hydrogen in the lattice and the circles represent the position of the saddle points. The solid lines show the network of minimum activation energy migration paths of hydrogen atoms in the tungsten lattice. By comparison with Figure 2 the strong modifications of the migration paths and connectivity patterns are clearly visible. Minima without associated migration paths are connected to saddle points outside of the displayed sample only. 


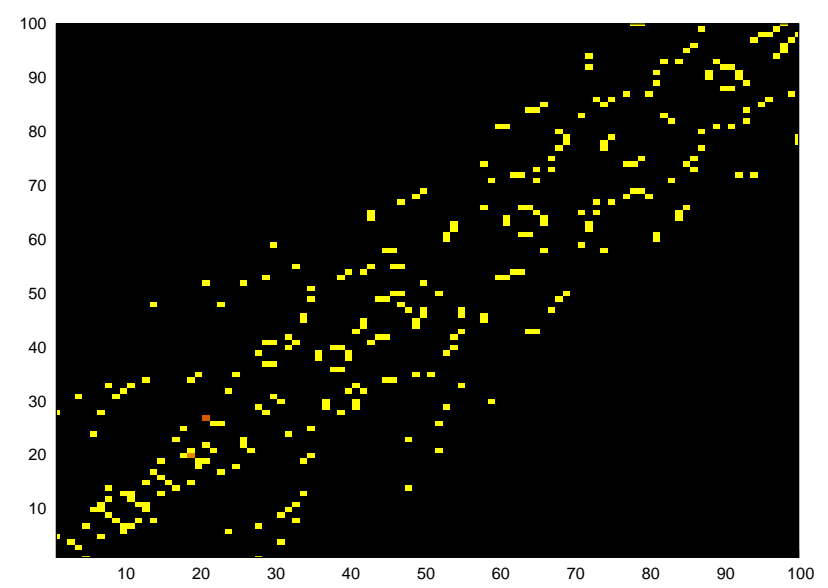

Figure 7. The connectivity matrix provides informations about the possible transport paths between individual minima. Each minimum has been assigned a label and the bright squares indicate to which other minima minimum activation energy migration paths exist. Please note that this matrix is symmetric (if there is a path from minimum $\mathrm{i}$ to $\mathrm{j}$ then also vice versa) in contrast to the transition probability matrix.

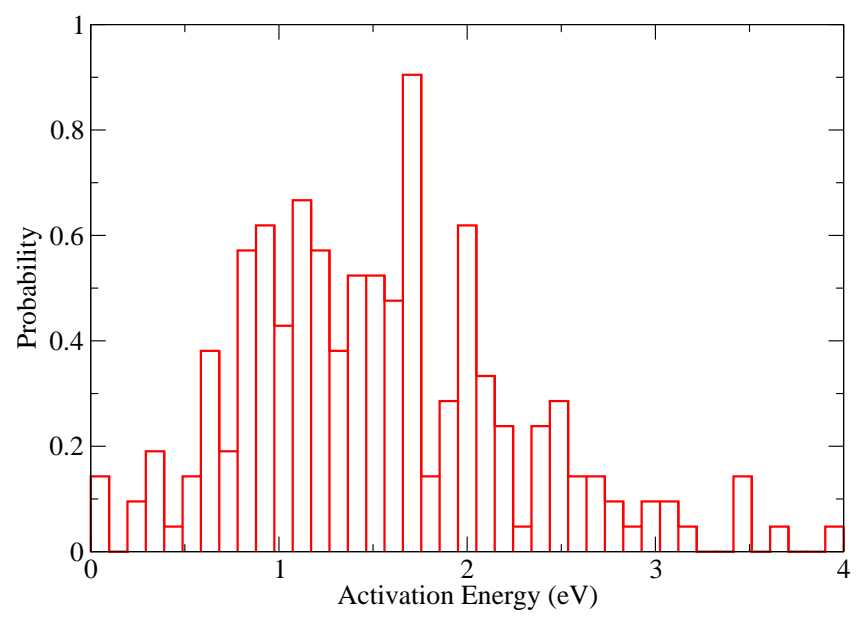

Figure 8. Distribution of the activation energies derived from all minimum activation energy migration paths. In a perfect bcc lattice the activation energies for the transition from a tetrahedral interstitial site to the next would all be identical (the MD potential yields $1 \mathrm{eV}$ ) but the distortion due to the grain boundary result in a much broader distribution, providing low activation energy migration paths. 